\title{
Low-pressure Chemical Vapour Deposition of Silicon Nanoparticles: Synthesis and Characterisation
}

\author{
A. Kumar ${ }^{1}$, Pankaj B. Agarwal ${ }^{1}$, Sachin Kumar ${ }^{2}$, B.C. Joshi ${ }^{1}$, A.K. Sharma ${ }^{1}$, and H. Chander ${ }^{3}$ \\ ${ }^{1}$ Central Electronics Engineering Research Institute, Pilani-333 031 \\ ${ }^{2}$ The Technological Institute of Textile and Sciences, Bhiwani-127 021 \\ ${ }^{3}$ National Physical Laboratory, New Delhi-110 012
}

\begin{abstract}
Semiconductor nanostructures such as quantum wells, quantum wires or quantum dots exhibit superior properties in comparison to their bulk forms. Quantum dots are described as zero-dimensional electron gas system, as carriers are confined in all the three directions. Density of states is discrete function of energy. Allowed energy spectrum is discrete like in an atom. Energy band gap is broadened due to carriers confinement. Semiconductor quantum dots exhibit typical coulomb blockade characteristic which is exploited for development of new generation of nanoelectronic devices namely single-electron transistor, memories, etc, whose operation depends on quantum mechanical tunneling of carriers through energy barriers. These semiconductor nanostructures emit light in visible range upon excitation by optical means. In recent years, research has been focused on different nano-scale materials; metals ( $\mathrm{Au}, \mathrm{Ag}, \mathrm{Fe}$, $\mathrm{Mn}, \mathrm{Ni})$, metal oxides $\left(\mathrm{SnO}_{2}, \mathrm{ZnO}_{2}\right)$, compound semiconductors (GaAs, GaAlAs, CdSe, CdS, $G a N$ ), and elemental semiconductors (silicon and germanium). As silicon is the most favoured material in the established integrated circuits manufacturing technology, research is being done for controlled synthesis and characterisation of $S i$ nanoparticles. The $S i$ nanoparticles have been synthesised on oxide and nitride layers over $S i$ substrate by IC technology compatible low-pressure chemical vapour deposition technique. Atomic force microscopy (AFM) characterisation has been extensively carried out on the samples. It is shown that the tip radius and shape of tip lead to less accurate estimate of the actual size. The AFM images have been evaluated based on the real surface topography and shape of the tip. Photoluminescence (PL) studies have been performed to characterise the samples. The PL measurements showed visible light emission from synthesised silicon nanoparticles.
\end{abstract}

Keywords: Silicon nanoparticles, coulomb blockade, quantum confinement, tip artifacts, convolution, deconvolution, surface reconstruction, single electron transistor, single electron memories

\section{INTROCUCTION}

Nanoscale materials exhibit interesting mechanical, optical, and electronic properties ${ }^{1}$. Metallic nanoparticles show extraordinary catalytic properties ${ }^{2}$, which are exploited in the synthesis of new materials and drug delivery ${ }^{3}$. Semiconductor nanostructures such as quantum well, quantum wire, and quantum dots have potential applications in various fields of nanoelectronics, photonics, photovoltaic, biomedicine, and biotechnology ${ }^{4-7}$. In semiconductor nanostructures, carriers are confined either in one- two- or all the three directions, and thus resulting in $2-, 1-$, or 0 - 
dimensional electron gas system, respectively. Besides, superior chemical and mechanical properties, semiconductor quantum dots exhibit discrete allowed energies spectrum like an atom. These structures exhibit unique characteristic called coulomb blockade. The coulomb blockade and quantum confinement are two basic phenomena, exploited to develop futuristic nanoelectronic devices such as resonant tunneling diode ${ }^{8}$ (RTD), single-electron transistor ${ }^{9}$ (SET) and single-electron memory ${ }^{10}$. In these singleelectron devices, it is possible to control the flow of charge with a precision of a single-electron. Due to quantum confinement, the band gap energy of quantum dots is increased. Visible light emission was discovered by Canham ${ }^{11}$ in porous silicon produced by electrochemical processes. Since then, extensive studies have been carried out on $\mathrm{Si}$ nanostructures for integrating opto-electronic devices along with CMOS chip. There are many techniques to synthesise Si nanoparticles, e.g., chemical ${ }^{12}$, mechanical ${ }^{13}$, chemical vapour deposition $(\mathrm{CVD})^{14}$, laser ablation ${ }^{15}$, scanning probe microscopy ${ }^{16}$, etc. Besides the synthesis process, their characterisation is extremely important. The most commonly used characterisation techniques are atomic force microscopy (AFM), x-ray diffraction (XRD), transmission electron microscopy (TEM), photoluminescence (PL), absorption spectroscopy, Raman spectroscopy and Fourier transform infrared spectroscopy (FTIR). Interpretation and manipulation of acquired AFM data from the sample to get accurate information is very important, for example, necessity of tip deconvolution of AFM images of very small nanoparticles.

The Si nanoparticles were synthesised by lowpressure chemical vapour deposition (LPCVD) ${ }^{17}$. The AFM imaging has been performed and images have been analysed using scanning probe image processor (SPIP) and WSxM software to study size, density, roughness, and distribution of nanoparticles ${ }^{18,19}$. Gwyddion 2.1 software has been used to study the effect of tip shape and size on the images ${ }^{20}$. In case of imaging the surfaces having feature with high aspect ratio, the obtained image is the result of the interaction between tip shape and sample surface. Due to this interaction, obtained image differs from the actual surface topography. To correct the image, it is necessary to have an estimate of the tip geometry, which is always not possible. Another method, for reconstruction of the surface topography without the prior knowledge of the tip shape, is the blind reconstruction method ${ }^{21-22}$.

\section{EXPERIMENTAL PROCEDURE}

\subsection{Synthesis}

The LPCVD process was used for synthesising $S i$ nanoparticles. Synthesis process has been described in details by Kumar, ${ }^{17}$ et al. $N$-type, $20 \Omega \mathrm{cm}$ resistivity, 2 " dia, $<100>$ orientation, $S i$ wafers were used in the experiments. After thorough chemical cleaning, an ultrathin $\mathrm{SiO}_{2}(8-10 \mathrm{~nm})$ was thermally grown at low temperature. On few wafers, thin silicon nitride $(\sim 2000 \AA)$ layer was deposited in LPCVD reactor. The Si nanoparticles were synthesised on these two types of $S i$ substrates by LPCVD process using pure silane $\left(\mathrm{SiH}_{4}\right)$ chemistry. Depositions were performed for very short duration, $30-90 \mathrm{~s}$ at temperatures in the range $550{ }^{\circ} \mathrm{C}-600{ }^{\circ} \mathrm{C}$. Several samples were prepared with different process parameters for the study.

\subsection{Characterisation}

Nano-dimensional structures are characterised by scanning probe microscopy(SPM) techniques, namely, AFM, scanning tunneling microscopy (STM). There are different modes of AFM imaging, namely contact, non-contact, intermittent contact (or tapping), and lateral force microscopy. Contact mode is the most common mode of operation of the AFM. In this mode, the tip and the sample remain in close contact (in the repulsive regime of the intermolecular force curve). One of the drawbacks of contact mode is that there exist large lateral forces on the sample as the tip is dragged over the specimen and may damage the sample of soft material like biological or polymer specimen. In non-contact mode, cantilever oscillates above the surface of the sample at such a distance that it is not in the repulsive force regime. This is a very difficult mode to operate in ambient conditions. The water meniscus, which exists on the surface on the sample, invariably forms a small capillary bridge between the tip and the sample and causes the tip to jump-to-contact. Tapping mode is the next most common mode used in AFM. When operated in air or other gases, the 
cantilever is oscillated at its resonant frequency (hundreds of $\mathrm{kHz}$ ) and is positioned above the surface so that it only taps the surface for a very small duration. As a result, lateral forces are significantly reduced as the tip scans over the surface. When imaging poorly immobilised or soft samples, tapping mode is a better choice than the contact mode. For this study, (nanosilicon/ $/ \mathrm{SiO}_{2} / \mathrm{Si}$ samples), the contact mode AFM imaging is employed as the sample material is hard and had gone through various high temperature cycles during fabrication. Digital Instrument DI-II AFM was used with scanner size of $1 \mu \mathrm{m}$.

The AFM images have been analysed using available software namely SPIP, WSxM, Gwyddion ${ }^{18-21}$. These software packages offer a wide variety of functions related to AFM image analysis including cross-section profile analysis, roughness analysis, grain/pore analysis, 3-D visualisation, plane correction (flattening), histogram analysis, Fourier transform, auto- and cross-correlation, tip characterisation, and deconvolution, zoom, noise reduction, etc. Line profiles were employed to estimate size and height of nanoparticles. Nanoparticle density has been calculated by counting particles in the window using the image processor.

Optical characterisation of few samples was carried out by PL measurements at room temperature on LS 55 system from Perkin Elmer. Xenon lamp was used as excitation source. The excitation wavelength was selected after observing the absorption spectra from the samples. Measurements were carried out in air at room temperature.

\section{RESULTS AND DISCUSSION}

The results of AFM imaging of $S i$ nanoparticles synthesised on $\mathrm{SiO}_{2} / \mathrm{Si}$ substrate at temperatures $575^{\circ} \mathrm{C}$ and $600{ }^{\circ} \mathrm{C}$ for different process durations of $30 \mathrm{~s}$ and $60 \mathrm{~s}$ are shown in Figs $1-3$.

The estimated particles size are in the range $5-17 \mathrm{~nm}$ with mean size $=10.6 \mathrm{~nm}$, and nanoparticles density $\sim 2.0 \times 10^{11} / \mathrm{cm}^{2}$ on sample processed at $575^{\circ} \mathrm{C}$ for $30 \mathrm{~s}$, while particle size are in the range $6-16 \mathrm{~nm}$ with mean size $=10.2 \mathrm{~nm}$ and nanoparticles density is $\sim 2.5 \times 10^{11} / \mathrm{cm}^{2}$ on sample fabricated at the same temperature but for $60 \mathrm{~s}$.
The 3-D view shows separated hemispherical nanoparticles on $\mathrm{SiO}_{2}$ surface. As the deposition time is increased, nucleation starts at new sites available. The images show nanoparticles of different sizes present on the samples. On samples synthesised at $600{ }^{\circ} \mathrm{C}$ for $30 \mathrm{~s}$ and $60 \mathrm{~s}$, estimated size of nanoparticles are in the range $6 \mathrm{~nm}-16 \mathrm{~nm}$ with mean size $=$ $10.4 \mathrm{~nm}$, and $6 \mathrm{~nm}-24 \mathrm{~nm}$ with mean size $=10.3 \mathrm{~nm}$ respectively. The estimated density of nanoparticles are $\sim 3.2 \times 10^{11} / \mathrm{cm}^{2}$ and $\sim 4.5 \times 10^{11} / \mathrm{cm}^{2}$ respectively for these samples. It was observed that nanoparticles density increased from $2.0 \times 10^{11} \mathrm{~cm}^{2}$ to $3.2 \times 10^{11} / \mathrm{cm}^{2}$ as deposition temperature was changed from $575{ }^{\circ} \mathrm{C}$ to $600{ }^{\circ} \mathrm{C}$ for $30 \mathrm{~s}$ deposition time.

Nucleation and growth is a random process. At a given temperature, growth on fresh nuclei starts and the existing ones grow in size. Nanoparticles density depends upon nucleation sites available for growth. Number of nucleation sites depends upon temperature and nature of surface. After increasingly long deposition time ( $>100 \mathrm{~s}$, in the present work), many nuclei collesced and formed large nanoparticles (or islands), thus, resulting in reduction in number of particles and increase in the average size. With further increase in deposition time, large islands further merged into a film. Surface defects, dangling bonds on the surface act as nucleation sites for incoming atoms of the material. On silicon nitride surface, there are more nucleation sites due to large number of unsatisfied bonds and higher surface roughness. A higher nanoparticles density may be expected on silicon nitride layer compared to the silicon dioxide layer with the same process parameters.

To verify this, the silicon nanoparticles were synthesised on $\mathrm{Si}_{3} \mathrm{~N}_{4} / \mathrm{Si}$ substrates through the above process. Figure 4 shows AFM images of $S i$ nanoparticles synthesised on samples with nitride surface layer at $575{ }^{\circ} \mathrm{C}$ for $60 \mathrm{~s}$. It is evident from the image that particles density at nitride surface layer is significantly higher than the oxide layer for the same process parameters. The estimated nanoparticles density is $\sim 5.0 \times 10^{11} / \mathrm{cm}^{2}$ and sizes are in the range $6-23 \mathrm{~nm}$ with mean size $10.4 \mathrm{~nm}$. This result indicates that the density of nanoparticles can be varied using rough surface or by intentional introduction of structural defects, dangling bonds, 


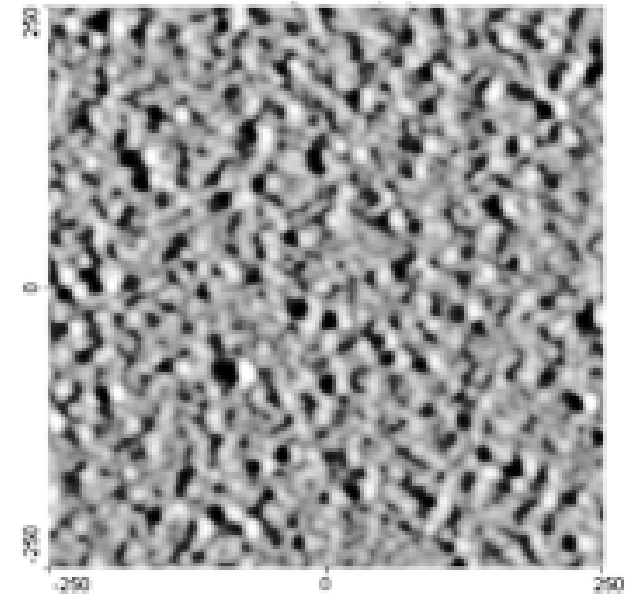

(a)

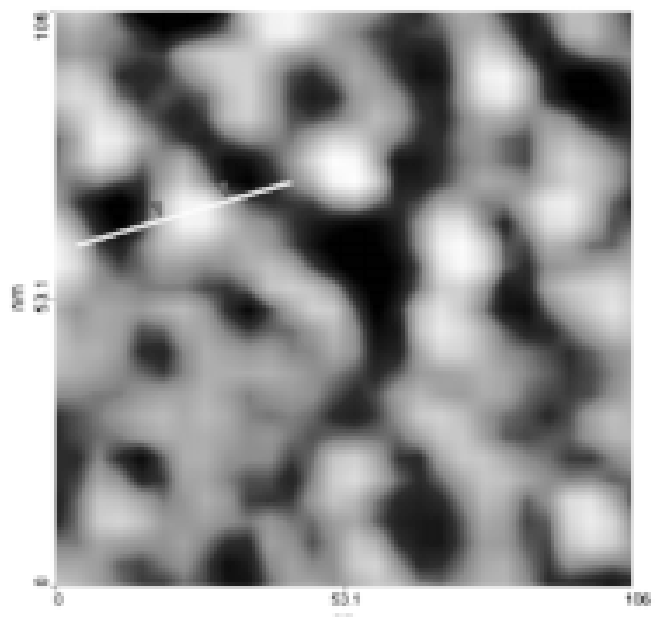

(c)

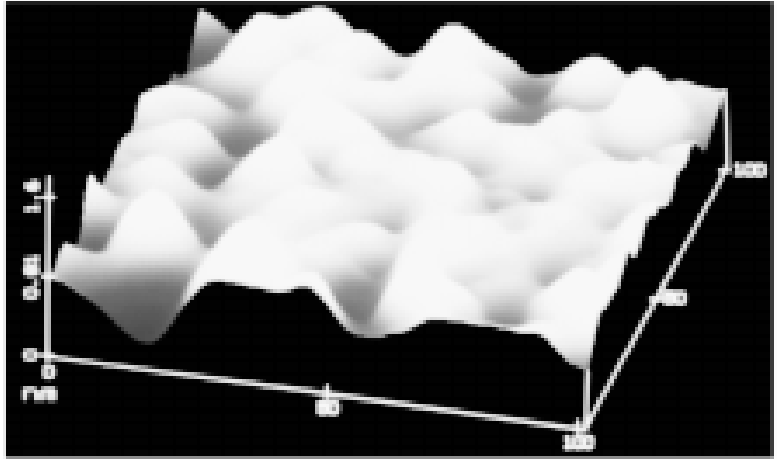

(b)

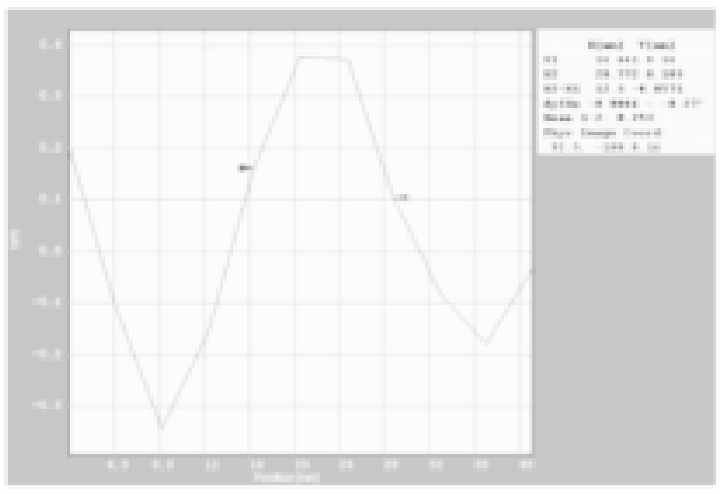

(d)

Figure 1. AFM image of the sample with $\mathrm{Si}$ nanoparticles deposited on $\mathrm{SiO} / \mathrm{Si}$ substrate at $575^{\circ} \mathrm{C}$ for $30 \mathrm{~s}$ : (a) top view; (b) 3-D view; (c) line profiling in the image; and (d) line profile along a nanoparticle.

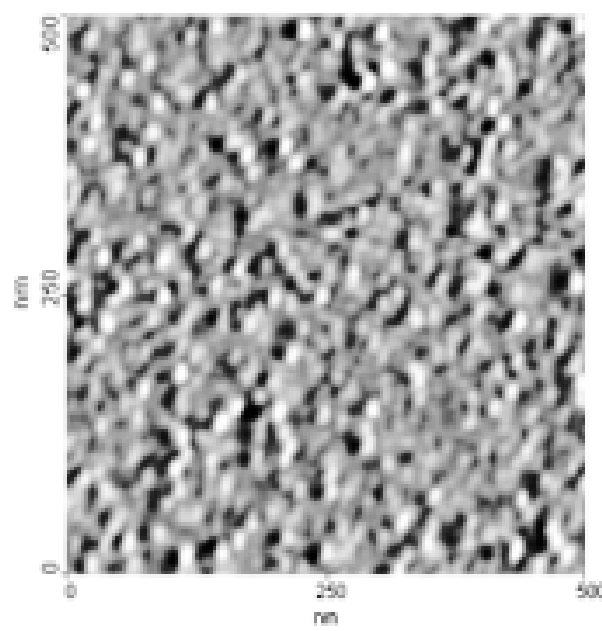

(a)

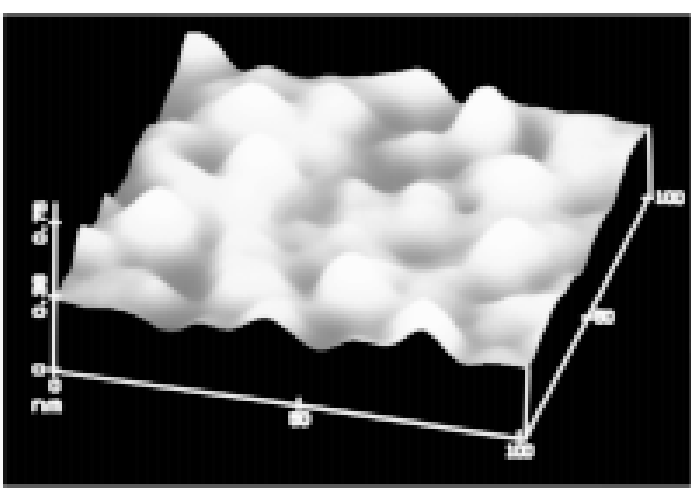

(b)

Figure 2. AFM image of the sample with $\mathrm{Si}$ nanoparticles deposited on $\mathrm{SiO} / \mathrm{Si}$ substrate at $575^{\circ} \mathrm{C}$ for 60 s: (a) top view; and (b) 3-D view. 


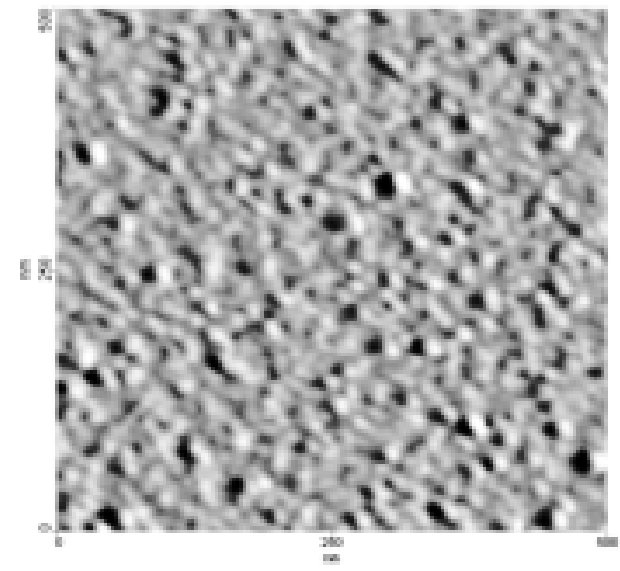

(a)

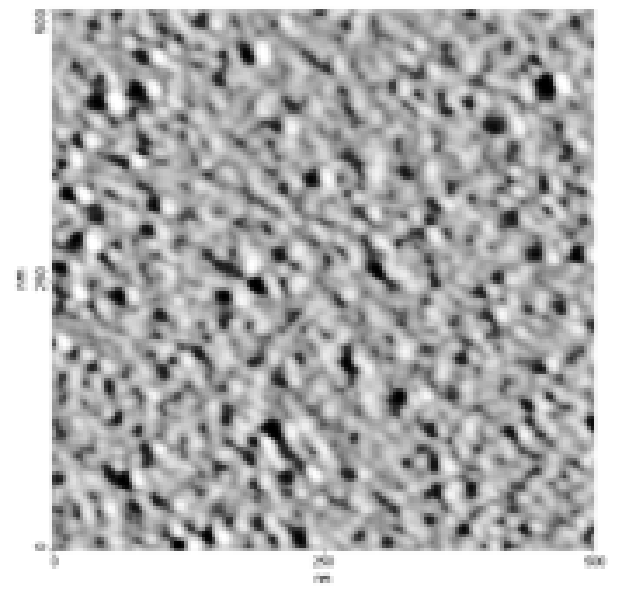

(b)

Figure 3. AFM images of the samples with $\mathrm{Si}$ nanoparticles deposited on $\mathrm{SiO}_{2} / \mathrm{Si}$ substrate at $600{ }^{\circ} \mathrm{C}$ for (a) $30 \mathrm{~s}$, and (b) $60 \mathrm{~s}$.

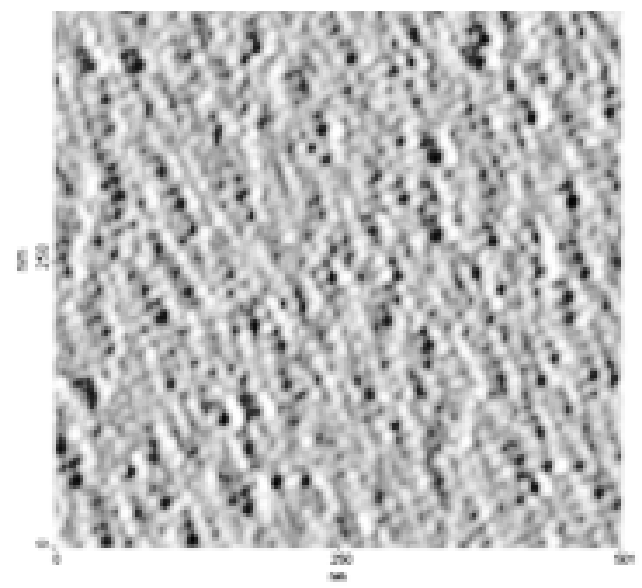

(a)

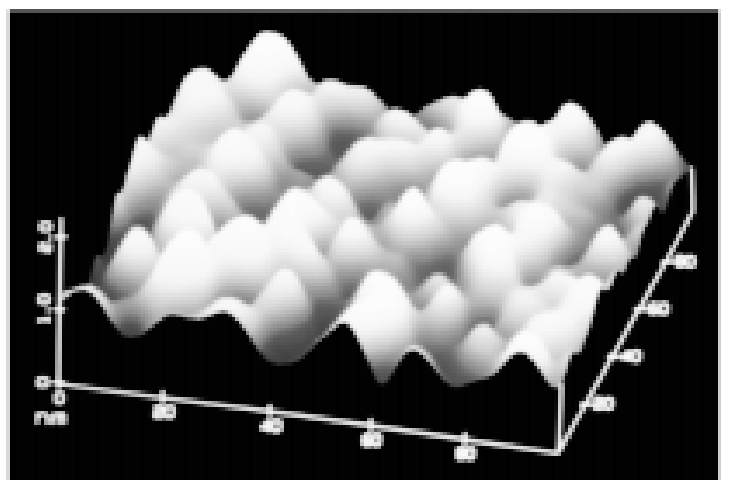

(b)

Figure 4. AFM image the sample with $\mathrm{Si}$ nanoparticles deposited on $\mathrm{Si}_{3} \mathrm{~N}_{4} / \mathrm{Si}$ substrate at $575^{\circ} \mathrm{C}$ for 60 s: (a) top view, and (b) 3-D view.

etc., in the under layer. Variation of nanoparticles density has been observed in experiments of $S i$ nanoparticles synthesis on oxy-nitride films ${ }^{25}$.

The PL spectra obtained from samples synthesised at $575{ }^{\circ} \mathrm{C}$ for $30 \mathrm{~s}$ (sample A1) and $60 \mathrm{~s}$ (sampleA2) are shown in Fig. 5. The observed PL spectra, from sample A1 showed emission peak in the visible range at $\lambda=627 \mathrm{~nm}$, and corresponding peak energy was $1.9757 \mathrm{eV}$. For sample A2, emission peak at $\lambda=629 \mathrm{~nm}$, corresponding peak energy was $1.9694 \mathrm{eV}$. The observed PL spectra showed blue shift of varying degrees on different samples. Emissions at $627.5 \mathrm{~nm}$ and $629.5 \mathrm{~nm}$ indicate that there are large number of nanocrystallites of few $\mathrm{nm}$ in size. On samples with long deposition times, i.e., $120 \mathrm{~s}$, no PL emission was detected.
The number of nuclei increases continuously with increase in the deposition time. During this nucleation and growth process, some smaller particles are annihiliated by large islands. These large islands do not show quantum confinement and light emission. The origin of light emission from $\mathrm{Si}$ nanoparticles is still debatable. In porous $\mathrm{Si}$, the light emission has been attributed to quantum confinement of carriers in the silicon nanowires ${ }^{11}$. Various mechanisms for light emission from $\mathrm{Si}$ nanoparticles have been put forward by different groups depending upon method of fabrication, dielectric matrix, passivation and impurities.

In the present work, the $S i$ nanoparticles were capped with the native oxide as the samples were exposed to the atmosphere. The visible light emission 
may be attributed to the quantum confinement in nanoscale $\mathrm{Si}$ cores. The oxide layer passivates the dangling bonds and sweeps out the energy states from the band gap. The Si nanoparticles particles contain lesser number of defects which act as killers or recombination centres for carriers. These and some other factors force the excited carriers to recombine through radiative process, thus, emitting photons. The emitted photons have energies corresponding to band gap of nanoparticles. The emission efficiency in $S i$ is extremely low due to its indirect band gap and recombination centres, in the energy gap.

In bulk $S i$, the excited carries are annihilated non-radiatively through these centres. These recombination centres are result of impurities, structural defects, and surface states, etc. If these structural defects are minimised, as in nanoparticles, the light emission from $S i$ is possible with improved efficiency.

\subsection{AFM Tip Convolution Effects and Methodology}

The AFM images are basically the results of tip-surface interaction. Radius of curvature of tip end, tip shape, and aspect ratio of the tip, dilate the actual information about features. The obtained image is called tip convoluted image. For example, a tip imaging a feature of negligible width would reveal the inverted geometry of the end of the tip, as shown in Fig. 6.

For better understanding of convolution, one may consider the surface as an ensemble of multiple thin columns and then collect inverted probes corresponding to all the columns (or positions). Now the SPM image is the superposition of envelops of all the inverted images. It can be easily seen from Fig. 7, that during AFM imaging there are tipshape features instead of actual spikes and also that there is no data corresponding to very small features due to its location on the substrate between the taller spikes.

To study how exactly this tip-sample interaction affects the image, one needs to reconstruct the surface. In case of surface reconstruction, data of original tip geometry is essential as the convolution is the product of two signals. In many cases, actual

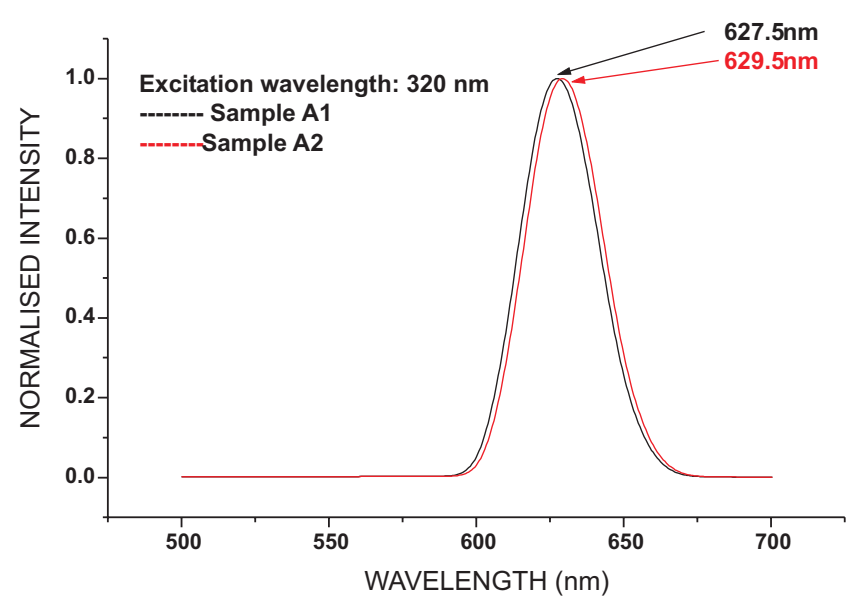

Figure 5. PL spectra of two samples deposited at $575^{\circ} \mathrm{C}$ for $30 \mathrm{~s}$ and $60 \mathrm{~s}$ on $\mathrm{SiO}_{2} / \mathrm{Si}$ substrates. Observed emission peaks are at $\lambda=627.5 \mathrm{~nm}$ and $629.5 \mathrm{~nm}$ in the visible range.

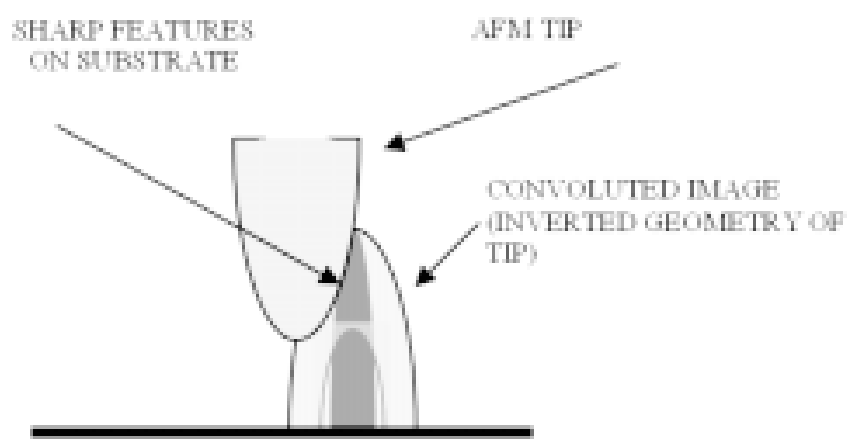

Figure 6. AFM imaging of sharp feature results in the inverted shape of AFM tip.

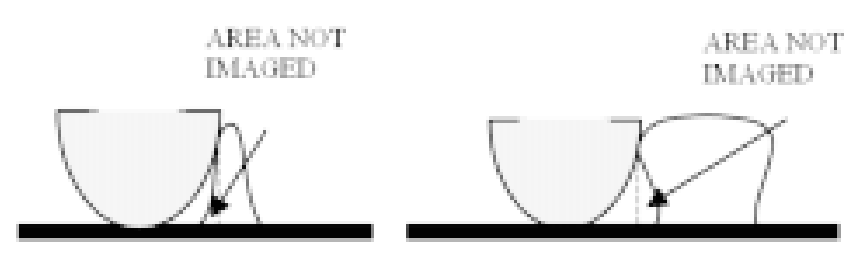

Figure 7. Tip dilation of structural features on the surfaces.

tip geometry is not known, in which case, it is desirable to reconstruct the surface using blind reconstruction algorithm.

The theoretical explanation and solution for tip convolution problem was given independently by Villarrubia $^{21}$ and Williams ${ }^{22}$, et al. Villarrubia ${ }^{23}$ also published the numerical implementation of the blind estimation algorithm. Some other experimental parameters like instrumental noise, sampling intervals, etc., which affect the AFM tip blind estimation, have been separately discussed by Tranchida, ${ }^{24}$ et 
al. Sometimes, it is important to find the area where the reconstruction is possible, using the certainty map. The present study analyses the AFM images to see the effect of tip convolution.

Figure 8 shows AFM image of the sample ( $S i$ nanoparticles) and the tip generated from the image data using Gwyddion 2.1. In this software, blind estimation method in run-fully mode is used to generate the tip. The height of generated tip is $\sim 0.4 \mathrm{~nm}$ and base dia $\sim 15 \mathrm{~nm}$. Deconvoluted image using generated tip is shown in Fig. 8 (b). Using the generated tip, the same image has been deconvoluted as shown in Fig. 9. The deconvoluted image gives better estimate about the shape and size of nanoparticles.
Window of size of $50 \mathrm{~nm} \times 50 \mathrm{~nm}$ has been selected to explain erosion process. Figures 10(a) and 10(b) compare the particles shape before and after deconvolution. Due to erosion process, as explained in Fig. 11, the sharp spikes appear in deconvoluted image. It has been observed that size of most of the particles is reduced by 30 per cent to 40 per cent, while maximum change in the height is 68 per cent. The reduction in size and height can be attributed to the erosion process during surface reconstruction and noise effects during imaging of the surface. The upper noise spikes have very little effect on the reconstructed surface compared to the lower noise spikes because during erosion process, only minimum envelope has been considered.

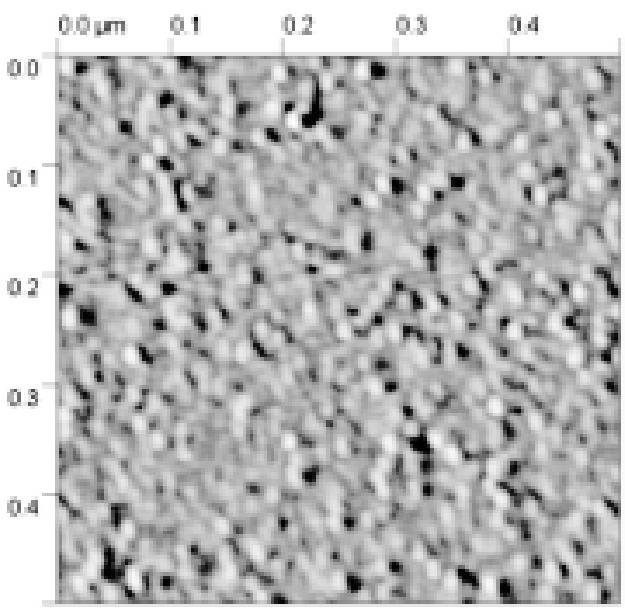

(a)

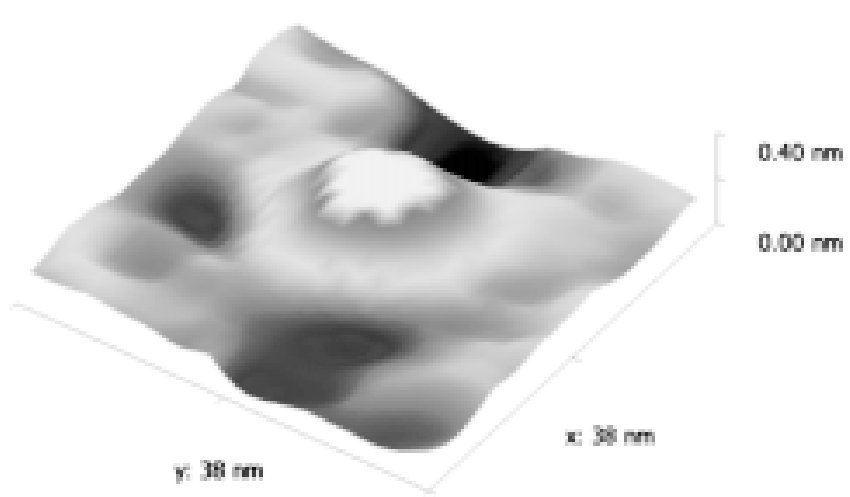

(b)

Figure 8. (a) Acquired AFM image of nanosilicon sample and (b) tip generated by blind estimation method using Gwyddion 2.1 software.

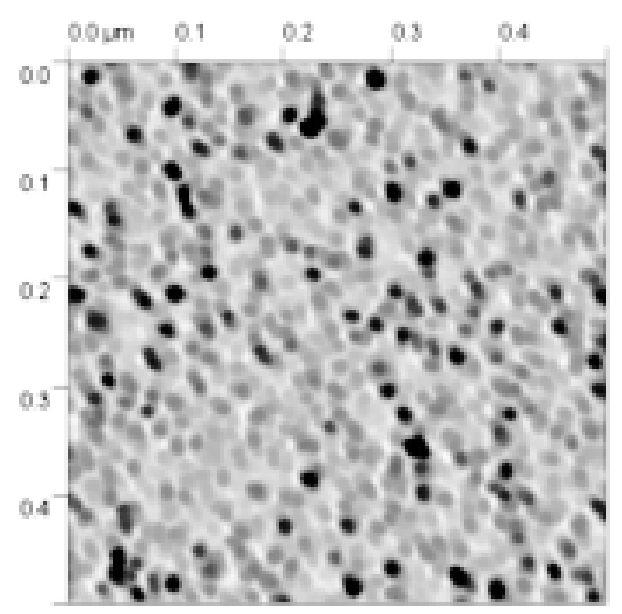

Figure 9. Image after tip deconvolution using generated tip in Fig. 8 (b).

\section{CONCLUSIONS}

The $S i$ nanoparticles have been synthesised on silicon substrate with oxide and nitride over layers. It has been demonstrated that nanoparticles density is higher $\left(5 \times 10^{11} / \mathrm{cm}^{2}\right)$ on the nitride surface compared to the oxide surface $\left(2.5 \times 10^{11} / \mathrm{cm}^{2}\right)$ for the same synthesis parameters. Detailed AFM image analysis has been presented for nano-particles size and density. Tip geometry has been generated from image data and deconvolution has been performed using Gwyddion 2.1 software. After deconvolution, the size of nanoparticles is reduced by 30 per cent to 40 per cent, while maximum change in height data of 68 per cent is observed. The PL has been observed from the synthesised $S i$ nanoparticles in 


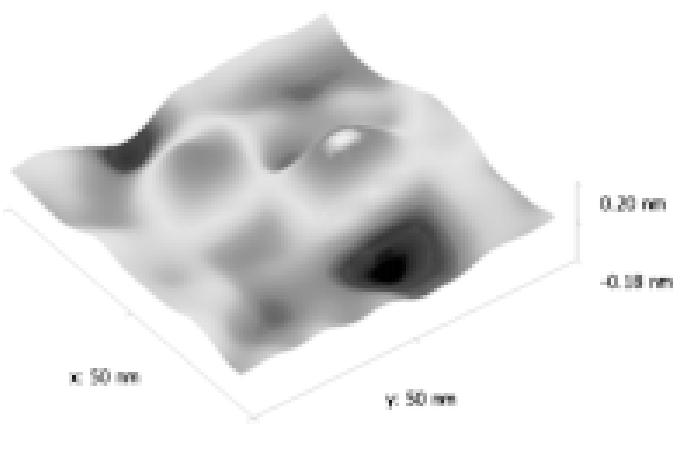

(a)

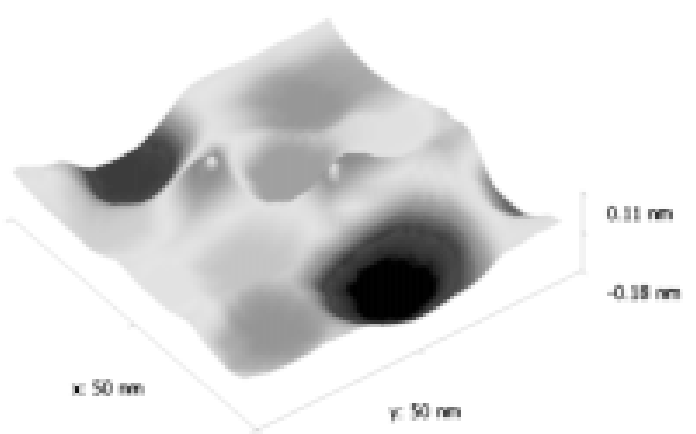

(b)

Figure 10. 3-D view of AFM image of $50 \mathrm{~nm} \times 50 \mathrm{~nm}$ window: (a) before tip deconvolution and (b) after tip deconvolution.

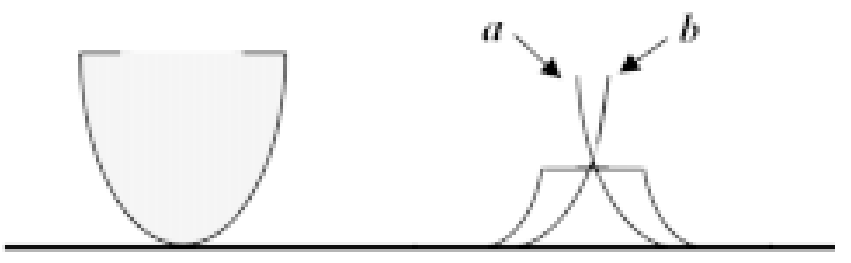

Figure 11. Erosion process of an image feature with a probe geometry that is too blunt. Erosion of right side of the image feature produces surface ' $a$ ' and erosion of left side of the image feature produces surface ' $b$ '.

the visible range.The PL results have been presented and possible mechanisms of emission have been discussed in detail. The Si nanoparticles have large number of potential applications in nanoelectronics, silicon photonics, memory, biotechnology, photovoltaics, drug delivery, etc.

\section{ACKNOWLEDGEMENTS}

This work has been carried out under a project sponsored by Department of Information Technology (DIT), New Delhi. The authors wish to thank Director, Central Electronics Engineering Research Institute (CEERI),Pilani, for his keen interest in the work. The authors also acknowledge the Semiconductor Devices Fabrication Facilities Group for fabrication support. Financial support from DIT is duly acknowledged.

\section{REFERENCES}

1. Bruchez, M.; Moronne, M.; Gin, P.; Weiss, S. \& Alivisatos, A.P. Semiconductor nanocrystals as fluorescent biological labels. Science, 1998, 281(5385), 2013-016.
2. Dossumov, K.; Salakhova, R.K.; Popova, N.M.; Tungatarova, S.A.; Grigorieva, V.P.; Komashko, L.U. \& Sass, A.S. Catalytical properties of nanostructured $P t$ and $R u$ metal clusters over alumina promoted by $\mathrm{CeO}_{2}$. J. Alloys Compounds, December 2006 (Online).

3. Suh, J.; Dawson, M. \& Hanes, J. Real-time multiple-particle tracking: applications to drug and gene delivery. Adv. Drug Deliv. Rev., 2005, 57(1), 63-78.

4. Heinrichsdorff, F.; Ribbat, C.; Grundmann, M. \& Bimberg, D. High-power quantum-dot lasers at $1100 \mathrm{~nm}$. Appl. Phys. Lett., 2000, 76(5), 556-58.

5. Henini, M. Properties and applications of quantum dot heterostructures grown by molecular beam epitaxy. Nanoscale Res. Lett., 2006, 1(1), 3245.

6. Eberl, K.; Lipinski, M.; Manz, Y. M.; Jin-Phillipp, N. Y. Winter, W.; Lange, C. \& Schmidt, O.G. Self-assembling InAs and InP quantum dots for optoelectronic devices. Thin Solid Films, 2000, 380(1-2), 183-88.

7. Clapp, A.R.; Medintz, I.L.; Mauro, J.M.; Fisher, B.R.; Bawendi,. M.G. \& Mattoussi, H. Quantum dots and peptides: A bright future together. $J$. Amer. Chem. Soc., 2004, 126, 301-10.

8. Reed, M.A.; Randall, J.N.; Aggarwal, R.J.; Matyi, R.J.;Moore,T.M. \& Wetsel, A.E. Observation of discrete electronic states in a zero-dimensional 
semiconductor nanostructure. Phys. Rev. Lett., 1988, 60, 535-37.

9. Devoret, M.H. \& Glattli, C. Single electron transistors.Physicsweb. September 1998. http://physicsweb.org/article/world/11/9/7.

10. Tiwari, S.; Rana, F.; Hussein, H.; Hartstein, A. $\&$ Crabbe, E.F. Silicon nanocrystals based memory. Appl. Phys. Lett., 1996, 68, 1377-379.

11. Canham, L.T. Silicon quantum wire array fabrication by electrochemical and chemical dissolution of wafers. Appl. Phys. Lett., 1990, 57, 1046048.

12. Rai, Akhilesh ; Chaudhary, Minakshi ; Ahmad, Absar.; Bhargava, Suresh \& Sastry, Murali. Synthesis of triangular $A u$ core- $A g$ shell nanoparticles. Mater. Res. Bull., 2007, 42(7), 1212-220.

13. Manova, E.; Tsoncheva, T.; Paneva, D.; Mitov, I.; Tenchev, K. \& Petrov, L. Mechanochemically synthesized nano-dimensional iron-cobalt spinel oxides as catalysts for methanol decomposition. Appl. Catalysis A: Gen., 2004, 277(1-2), 11927.

14. Baron,T.; Mazen, F.; Busseret, C.; Souifi, A.; Mur, P. ; Fourrel, F.; Siemria, M.N.; Moriceau, H.; Asparel, B.; Gentile, P. \& Magnea, N. Nucleation control of CVD growth silicon nanocrystals for quantum devices. Microelectronics Engineering, 2000, 61-62, 511-15.

15. Uchida, N.; Okami, T.; Tagami, H.; Fukata, N.; Mitome, M.; Bando, Y. \& Murakami, K. Synthesis of silicon nanocrystals in aluminumdoped $\mathrm{SiO}$ film by laser ablation method. Physica E: Low-dimensional Sys. Nanostruct., 2007, 38(1-2), 31-35.
16. Xie, X.N.; Chung, H.J.; Sow, C.H. \& Wee, A.T.S. Nanoscale materials patterning and engineering by atomic force microscopy nanolithography. Mater. Sci. Engg., 2006, 54(12), 1-48.

17. Kumar, A.; Joshi, B. C.; Sharma, A. K.; Karthigeyan, A.; Runthala, D.P. \& Gupta, R.P. Synthesis and characterisation of silicon nanoparticles by chemical vapour deposition for nanoelectronic devices applications. In Proceedings of $13^{\text {th }}$ International Workshop on Physics of Semiconductor Devices (IWPSD-2005), Vol.1. Allied Publishers Pvt Ltd., 2005. pp.319-22.

18. The Scanning Probe Image Processor, SPIP ${ }^{\mathrm{TM}}$. www.imagemet.com,

19. WSxM 2.2 Scanning Probe Microscopy Software. www.nanotec.es

20. Gwyddion 2.1 SPM data analysis framework. http://gwyddion.net.

21. Villarrubia, J.S. Morphological estimation of tip geometry for scanned probe microscopy. Surface Science, 1994, 321, 287-300.

22. Williams, P.M.; Shakesheff, K.M.; Davies, M. C.; Jackson, D.E.; Roberts, C.J. \& Tendler, S. J.B. Blind reconstruction of scanning probe image data. J. Vacuum Sci. Technol., B, 1996, 14, 1557-562.

23. Villarrubia, J.S. Algorithms for scanned probe microscope image simulation, surface reconstruction, and tip estimation. J. Res.Nat.Inst. Standards Technol., 1997, 102(4), 425-54.

24. Tranchida, D.; Piccarolo, S. \& Deblieck, R. A.C. Some experimental issues of AFM tip blind estimation: The effect of noise and resolution. Measurement Sci. Technol., 2006, 17, 2630636. 